\title{
Uma Abordagem para Medição e Análise em Projetos de Desenvolvimento de Software
}

\author{
Lílian Schnaider, Gleison Santos, Mariano Montoni, Ana Regina Rocha \\ Universidade Federal do Rio de Janeiro \\ Caixa Postal 68511 CEP 21945 -970 Rio de Janeiro - RJ, Brasil \\ \{schnaider, gleison, mmontoni, darocha\}@cos.ufrj.br
}

\begin{abstract}
Resumo
Durante o processo de Medição e Análise é desenvolvida e implantada a capacidade de avaliação das medidas que servirão como importantes fontes de informação para a gerência da Organização. A melhor abordagem para seleção das medidas adequadas é a que define, antes de tudo, o que a Organização deseja ou precisa saber, e somente então escolhe as medidas apropriadas. Este artigo apresenta uma abordagem para o processo de Medição e Análise em projetos de desenvolvimento de software baseada no método Goal-Question-Metrics (GQM) e nos requisitos da área de processo Medição e Análise do CMMI. Apresenta também duas ferramentas de apoio à abordagem proposta.

Palavras-chave: Medição e Análise, Qualidade de Software, Ambiente de Desenvolvimento de Software
\end{abstract}

\begin{abstract}
During the Measurement and Analysis Process is developed and implemented the capacity to evaluate the metrics that will be important sources of information to Organization's managers. The best approach to select the appropriate metrics is the one that, first of all, defines what the Organization whishes or needs to know, and only after this selects the adequate metrics. This article describes an approach to Measurement and Analysis Process for software projects based on the Goal-Question-Metrics (GQM) method and the CMMI Measurement and Analysis Process Area. It also presents two tools that support this approach.
\end{abstract}

Keywords: Measurement and Analysis, Software Quality, Software Development Environment

\section{Introdução}

Processos de medição se tornaram uma parte tão importante quanto necessária nas Organizações que desenvolvem software [8], pois para competir em um ambiente caracterizado por rápidas e constantes mudanças, é fundamental trabalhar de maneira produtiva, eficiente e com alto nível de qualidade. Por estes motivos, os dias de tomadas de decisão baseadas apenas "em palpites" terminaram, e é neste contexto que a medição se insere: a partir da existência de dados e análises históricas sobre a Organização é possível melhorar em muito o processo de tomada de decisão [7].

A maioria dos profissionais da área de desenvolvimento de software compreende a necessidade de se realizar medições, mas, infelizmente, a implementação de um processo que venha a se tornar repetível e integrado aos ciclos-de-vida de desenvolvimento e manutenção de software de uma forma geral ainda é um grande problema. As principais razões para o fracasso de programas de medidas não são problemas técnicos, e sim organizacionais [11], tais como: não-alinhamento aos objetivos de negócio, resistência cultural, motivação errônea e falta de liderança. De acordo com Holmes [5], estes problemas problemas podem ser resolvidos abordando-se a definição e implementação de um processo de medição através do uso de uma metodologia organizada de planejamento, que busca envolver todos os profissionais da área de software, cada um no momento adequado.

Um programa de medidas de sucesso é mais que simplesmente uma coletânea de dados. Os benefícios e valores agregado obtidos através da medição dizem respeito às decisões e ações tomadas a partir da análise dos dados obtidos, e não da coleção de dados em 
si [15]. Portanto, a melhor abordagem para definição e implementação de um processo de medição é a que define, antes de tudo, o que a Organização deseja ou precisa saber, e somente então escolhe as medidas apropriadas. Uma vez que as medidas estejam definidas o passo seguinte é encontrar uma coleção de dados específica que possa apoiar a obtenção destas medidas. Especificamente, um processo deste tipo envolve os seguintes passos: (i) definir os objetivos e iniciativas; (ii) definir as medidas que apoiarão estes objetivos e iniciativas, (iii) definir os dados que serão necessários para produzir estas medidas; (iv) definir como analisar e comunicar os resultados das medidas e (v) implementar o processo [5].

Um processo de medição de software direcionado aos objetivos produz medidas que provêm informações para importantes questões de negócio previamente identificadas. Uma vez que as medidas podem ser rastreadas de volta aos objetivos da Organização, as atividades de coleta de dados não são executadas apenas pelo ato de coletar medidas, e sim com o propósito de que os dados coletados sejam analisados de forma a manter o foco nestes objetivos $[4,5]$.

O método Goal-Question-Metrics (GQM) proposto por Basili [2] é um método de medição direcionado a objetivos, e tem sido adotado para medir e melhorar a qualidade em Organizações de desenvolvimento de software. O modelo de medida proposto por este método contém três níveis: conceitual (objetivos), operacional (questões) e quantitativo (métricas). Objetivos são definidos para um objeto (produto, processo, ou recurso utilizado por um processo), questões definem caminhos para se alcançar um determinado objetivo, e métricas associam dados às questões para que se possa respondê-las de forma quantitativa.

O GQM é composto das seguintes fases:

(i) planejamento, que envolve a seleção da aplicação a ser mensurada, e a definição, caracterização e planejamento do projeto;

(ii) definição, onde os objetivos, questões, métricas e hipóteses são definidas e documentadas;

(iii) coleta de dados, para atender as métricas definidas; e

(iv) interpretação, na qual os dados coletados são analisados para identificar as respostas às questões [12].

Ambientes de Desenvolvimento de Software Orientados à Organização (ADSOrg) se propõem a apoiar a atividade de Engenharia de Software, possibilitando a gestão do conhecimento que pode ser útil aos engenheiros de software ao longo dos projetos de uma Organização [13]. Desta forma, o conhecimento dos objetivos, questões e métricas de uma Organização é um dos conhecimentos que devem ser gerenciados por este tipo de ambiente. Este artigo apresenta uma abordagem para a medição e análise de processos e produtos de software inserida no contexto de Ambientes de Desenvolvimento de Software Orientados à Organização. A proposta é apoiar a medição e análise disponibilizando o conhecimento sobre medições que possa ser útil às Organizações e aos gerentes de projeto durante a execução das atividades que compõem o processo de Medição e Análise.

A seção seguinte enfoca a área de processo de Medição e Análise do CMMI [16], a qual estabelece requisitos para o processo de Medição e Análise definido com base na literatura e apresentado na seção 3 deste artigo. A seção 4 discute a abordagem proposta para a medição e análise, apresentando as ferramentas MedPlan e Metrics e, finalmente, a seção 5 apresenta as considerações finais.

\section{A Área de Processo Medição e Análise no CMMI}

Várias abordagens para medição de software existem hoje em dia: vários padrões internacionais foram publicados e existe muita literatura relacionada a medição de software e 
métricas. Entretanto, existe também uma comunidade emergente que pratica e divulga a combinação entre medição de software e melhorias em processos - comunidade esta que trabalhou de maneira colaborativa com colegas do Practical Software and Systems Measure Support Center no desenvolvimento da área de processo de Medição e Análise do Capability Maturity Model Integration (CMMI) [16] e no desenvolvimento do padrão ISO relacionado à medição de software e à avaliação de processos [6].

A área de processo de Medição e Análise foi um importante adendo ao CMMI. Seu escopo é bem mais abrangente e explícito que o tratamento dispensado à questão de medição no Capability Maturity Model for Software (SW-CMM) [10]. O modelo CMMI-SE/SW/IPPD possui 24 áreas de processo que encontram-se agrupadas em quatro categorias: gerência do processo, gerência do projeto, engenharia e suporte [1].

O propósito da área de processo de Medição e Análise, pertencente à categoria de suporte do CMMI, é desenvolver e manter a capacidade de medição que será utilizada para apoiar as necessidades de informações gerenciais da Organização. Esta área de processo provê um guia essencial a ser seguido sempre que houver necessidade de medição, sendo que suas práticas são sempre executadas dentro do contexto de execução de outros processos [6]. Se considerado o CMMI em estágios, esta é uma área de processo de nível 2.

A área de processo de Medição e Análise possui dois objetivos: (i) alinhar as atividades relacionadas à medição às necessidades informacionais da Organização, e (ii) fornecer os resultados das medições de forma a satisfazer a estas necessidades de informação. Para atingir ao primeiro objetivo a equipe responsável pelas medições estabelece os objetivos de medição da Organização, e especifica as métricas e os procedimentos de coleta, armazenamento e análise de dados. Para atingir ao segundo objetivo deve-se coletar os dados e os resultados das medições, armazená-los, analisá-los e comunicar os resultados aos interessados. A Figura 1 apresenta a área de processo de Medição e Análise [1].

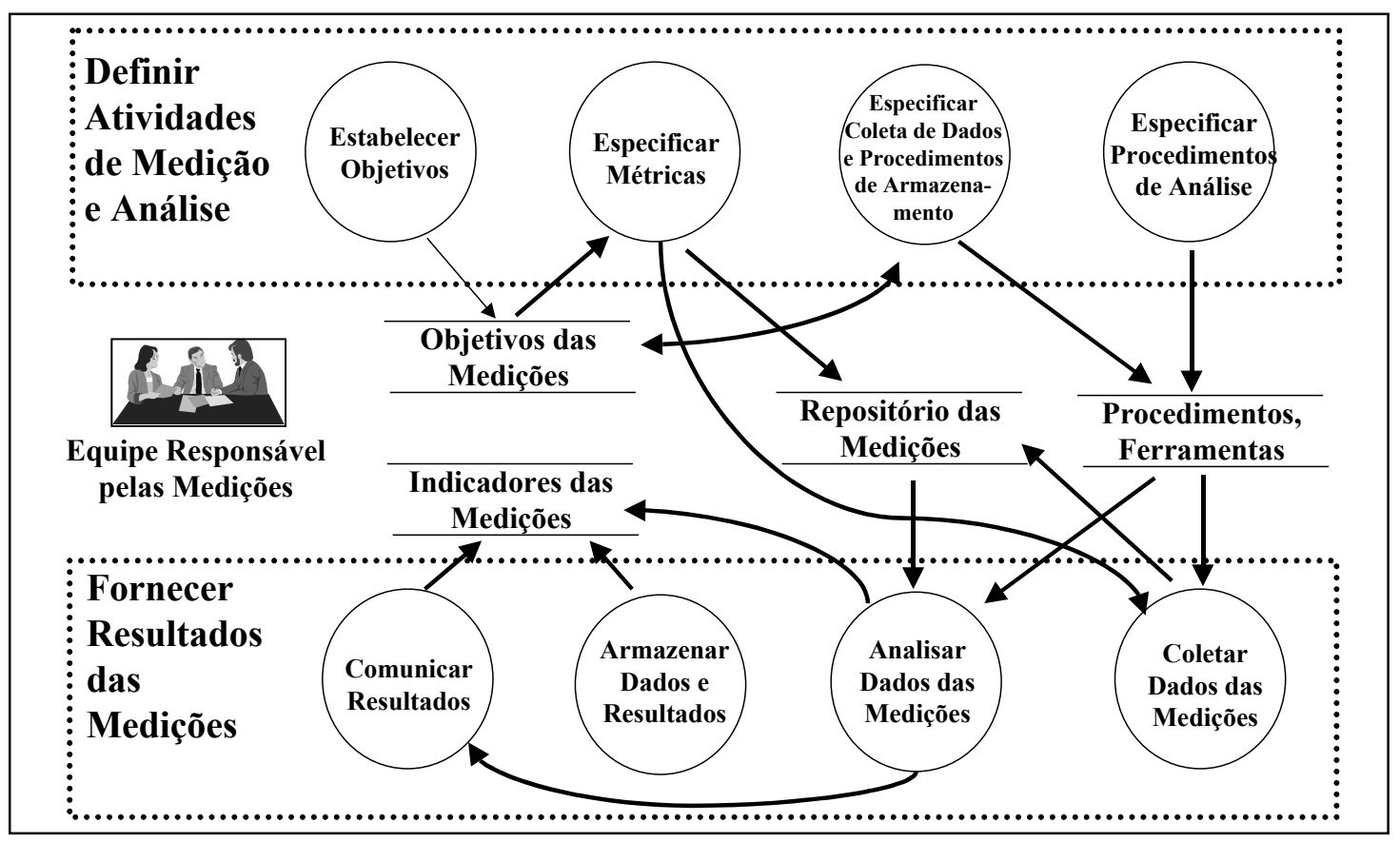

Figura 1 - Área de Processo de Medição e Análise [1] 
As práticas específicas associadas ao objetivo (i) - "alinhar as atividades relacionadas à medição às necessidades informacionais da Organização" - estabelecem um plano coerente para a medição e análise (Plano de Medição da Organização), endereçando questões tais como "o que estamos medindo?", "porque estamos medindo?", "como vamos medir?" e "o que vai ser feito com os dados e resultados, uma vez que os tenhamos?". Já as práticas específicas associadas ao objetivo (ii) - "fornecer os resultados das medições de forma a satisfazer a estas necessidades de informação" - apenas direcionam o usuário para fazer o que tem que ser feito [6]. O objetivo maior, resumindo, é "colocar os resultados das medições e análises nas mãos daqueles que vão tomar decisões e ações com base nestes dados. O que esta área de processo do CMMI enfatiza é a necessidade de que os resultados obtidos sejam adequadamente comunicados aqueles que realmente precisam da informação" [14].

Através de uma abordagem que fornece os resultados dos objetivos de medição, esta área de processo propicia a execução das ações corretivas apropriadas, uma vez que a tomada de decisões é feita com base em informações reais e históricas da própria Organização.

Tal como as demais áreas de processo, a de Medição e Análise pode progredir desde o status de estar sendo executada de maneira "ad-hoc" até o seguimento do fluxo de um processo bem definido, que se utiliza de medidas para avaliar e melhorar seu próprio desempenho [6]. O processo de Medição e Análise apresentado a seguir se propõe a ser este último tipo de processo.

\section{O Processo de Medição e Análise}

De acordo com Florac e Caleton [3], um processo de medição deve ser modelado de forma independente do processo de desenvolvimento, e conter atividades para coleta de métricas que contenham a descrição de cada métrica assim como uma descrição clara de seus objetivos. $\mathrm{O}$ processo de Medição e Análise definido atende à [3] e aos requisitos da área de processo de Medição e Análise do CCMI [16].

O objetivo do processo de Medição e Análise é devenvolver e implantar a capacidade de avaliação das medidas (métricas) que servirão como importantes fontes de informação para a gerência da Organização. É importante ter em conta que os benefícios obtidos na realização das medições vêm das decisões e ações tomadas a partir da análise dos dados e não, apenas, da ação de se coletar dados. A Figura 2 descreve o processo de Medição e Análise definido.

Vários artefatos são produzidos ao longo da execução do processo proposto, dentre eles o Plano de Medição da Organização, o Documento de Especificação das Métricas e os Relatórios de Análises dos Resultados, sendo que o gerente de informática da Organização é o responsável pela elaboração da maior parte destes artefatos. Deve-se enfatizar que as saídas de cada sub-atividade representam as entradas da sub-atividade subsequente, seguindo-se a ordem em que aparecem no processo proposto.

A proposta do processo de Medição e Análise definido é então apoiar as necessidades de medição e análise da Organização como um todo, possibilitando:

(i) a elaboração do Plano de Medição a partir dos objetivos de informação definidos pela Organização, e

(ii) o fornecimento dos resultados das medições estabelecidas. A seguir são apresentadas as ferramentas MedPlan e Metrics, cujos objetivos, por sua vez, são apoiar o processo definido. 


\section{PROCESSO DE MEDIÇÃO E ANÁLISE}

Atividade: $\quad$ Especificar Atividades de Medição e Análise

Descrição: Identificar objetivos e práticas para medições de forma que estes estejam alinhados às necessidades e objetivos de informação da Organização. A identificação de objetivos, questões e métricas é feita utilizando-se a abordagem GQM (Goal-Question-Metrics) de Basili.

Sub-Atividades:

Nome: $\quad$ Identificar Objetivos de Medição

Descrição: Estabelecer objetivos para medições derivados das necessidades e objetivos de informação da Organização.

Nome: $\quad$ Identificar Questões de Medição

Descrição: $\quad$ Estabelecer questões a serem respondidas para que os objetivos de medição da Organização possam ser mensurados.

Nome: $\quad$ Identificar Medidas

Descrição: $\quad$ Especificar medidas (métricas) para avaliar o atingimento dos objetivos definidos.

Nome: $\quad$ Especificar Procedimentos de Coleta e Armazenamento de Dados

Descrição: $\quad$ Especificar como os dados de medições serão obtidos e armazenados

Nome: $\quad$ Especificar Procedimentos de Análise

Descrição: $\quad$ Especificar como os dados de medições serão analisados e reportados

Atividade: $\quad$ Fornecer Resultados das Medições

Descrição: Obter e fornecer os resultados das medições realizadas de acordo com as necessidades e objetivos de informação identificados pela Organização

\section{Sub-Atividades:}

Nome:

Descrição:

Nome:

Descrição:

Nome:

Descrição:

Nome:

Descrição:

\section{Coletar Dados de Medições}

Obter os dados das medições de acordo os procedimentos especificados para coleta, garantindo sua integridade

Analisar Dados de Medições

Analisar e interpretar os dados obtidos nas medições

\section{Armazenar Dados e Resultados}

Gerenciar e armazenar os dados obtidos nas medições assim como as especificação das medições e os resultados de análise.

\section{Comunicar Resultados}

Comunicar os resultados das medições e das atividades de análise a todos os envolvidos. 


\section{As Ferramentas MedPlan e Metrics}

Buscando-se apoiar a abordagem de medição e análise descrita neste trabalho, as ferramentas MedPlan e Metrics foram definidas e implementadas. Ambas as ferramentas são diponibilizadas em ADSOrg e, desta forma, possibilitam a utilização do conhecimento de medição e análise armazenados nos repositórios do ambiente.

\subsection{A Ferramenta MedPlan}

A proposta da ferramenta MedPlan é apoiar a elaboração dos Planos de Medição da Organização e do Projeto. Baseando-se no método GQM, esta ferramenta disponibiliza ao usuário o conhecimento sobre objetivos, questões, métricas e procedimentos de coleta, armazenamento e análise de dados a serem utilizados.

O Plano de Medição deve ser elaborado primeiramente para a Organização como um todo, e neste deverão estar definidos os objetivos corporativos de medição, as questões a serem respondidas para que se possa avaliar o atingimento destes objetivos e as medidas (métricas) propriamente ditas (assim como seus procedimentos de coleta, armazenamento e análise de dados) que serão utilizadas para responder às questões formuladas.

Uma vez elaborado o Plano de Medição da Organização seus itens farão parte do Plano de Medição de cada Projeto desta mesma Organização. O gerente de projeto pode, se desejar, acrescentar (nunca subtrair) novos objetivos, questões ou métricas que serão específicos do projeto em questão. Para tal, a ferramenta disponibiliza para o gerente de projeto o Plano de Medição da Organização, assim como o conhecimento do ambiente a respeito de objetivos de medição e suas respectivas questões e métricas associadas.

Para apoiar as atividades descritas a ferramenta MedPlan:

(i) fornece um mecanismo de planejamento de medições no nível corporativo e também no nível de cada projeto, utilizando como base a gerência do conhecimento organizacional;

(ii) auxilia a execução do processo de Medição e Análise da Organização, através da disponibilização de objetivos, questões e métricas parametrizadas;

(iii) auxilia o gerente de projeto no planejamento de medições para o seu projeto através da disponibilização do Plano de Medição da Organização, da disponibilização de objetivos, questões e métricas parametrizadas, e do conhecimento acumulado em projetos anteriores; $\mathrm{e}$,

(iv) apoia o acompanhamento e evolução do Plano de Medição ao longo de cada projeto.

A Figura 3 apresenta a interface básica da ferramenta MedPlan. No lado esquerdo da interface pode-se identificar a atividade "Elaborar Plano de Medição da Organização" e suas respectivas sub-atividades, e no lado direito identifica-se a sub-atividade que está sendo realizada pelo usuário. Especificamente, a Figura 3 apresenta a sub-atividade "Definir Plano de Medição da Organização". 


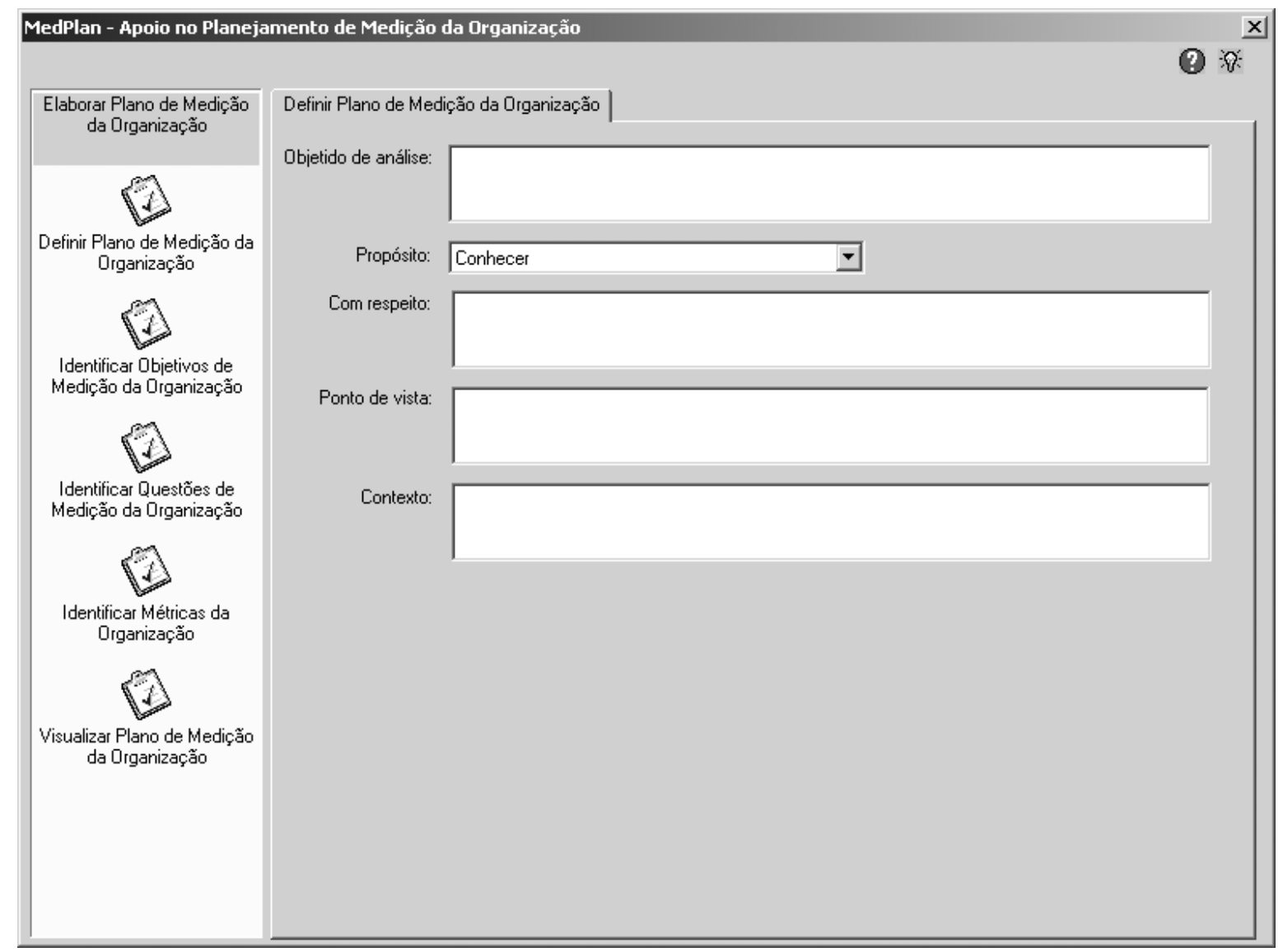

Figura 3 - Interface Básica da Ferramenta MedPlan

Os ícones localizados abaixo da barra de título permitem a busca e o registro de conhecimento no que diz respeito às atividades do processo. $\mathrm{O}$ gerente do projeto pode consultar idéias e lições aprendidas registradas por gerentes de projetos anteriores e também pode registrar suas próprias idéias e lições. A MedPlan disponibiliza também o conhecimento explícito em relação ao processo de Medição e Análise através de interface com a ferramenta de Aquisição do Conhecimento Acknowledge [9].

Na sub-atividade "Definir Plano de Medição da Organização" o usuário define pontos tais como o propósito das medições a serem realizadas (melhorar ou conhecer a Organização, por exemplo) e o contexto em que o Plano será elaborado. Em "Identificar Objetivos da Organização" o usuário seleciona os objetivos a serem alcançados pelas medições a serem efetuadas na Organização. Para cada objetivo identificado o usuário deve selecionar uma ou mais questões que devem ser respondidas para seu atingimento na subatividade "Identificar Questões da Organização". Na atividade "Identificar Métricas da Organização", por sua vez, o usuário deve selecionar uma ou mais métricas a serem utilizadas para responder a cada uma das questões previamente identificadas. Para cada métrica apresentada a ferramenta informa seus respectivos objetivos, descrições e procedimentos de coleta, armazenamento e análise. A figura 4 apresenta a tela da sub-atividade "Identificar Métricas da Organização”. 


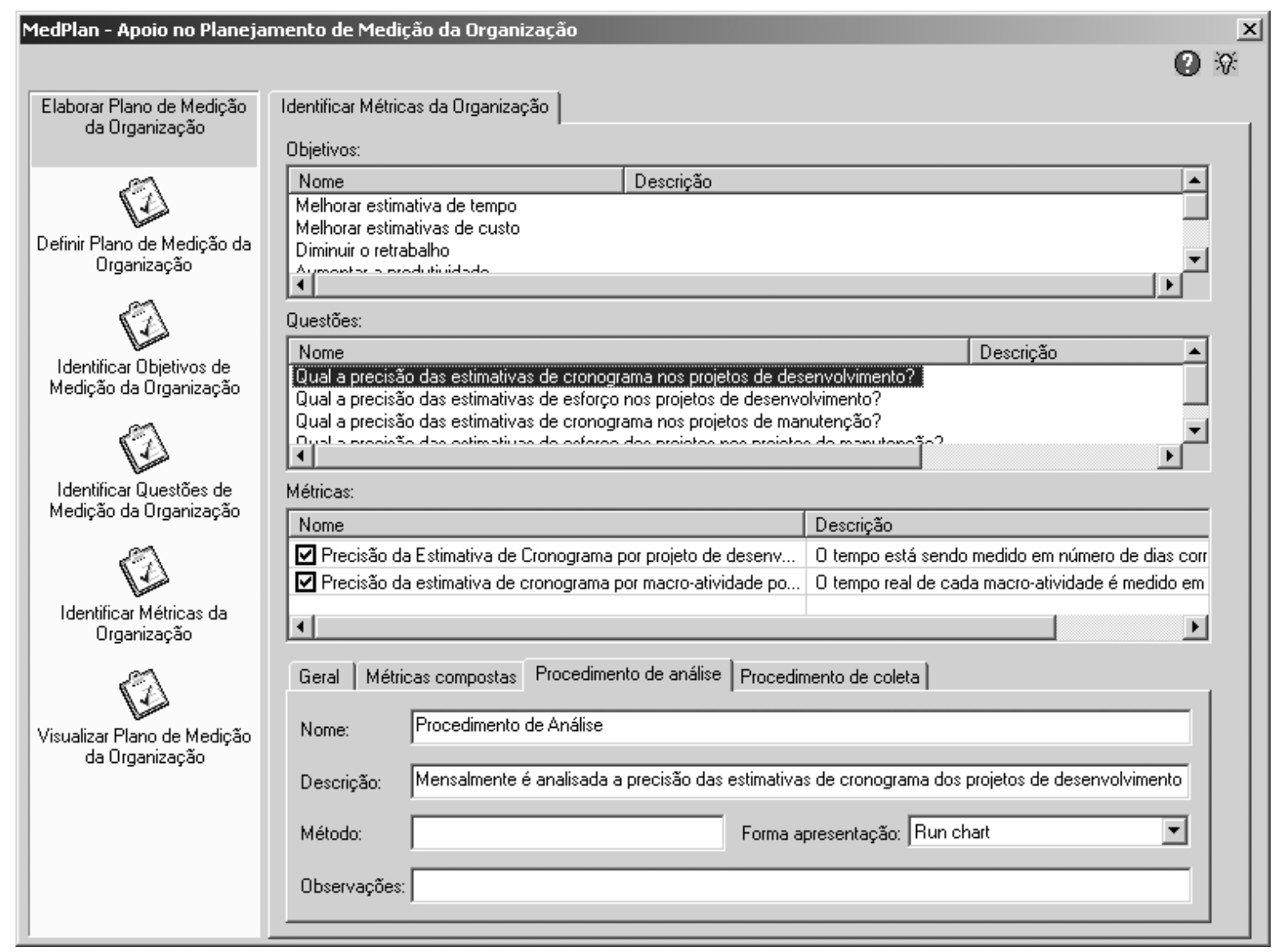

Figura 4 - Tela da sub-atividade "Identificar Métricas da Organização"

No Plano de Medição da Organização gerado com o auxílio da ferramenta na subatividade "Visualizar Plano de Medição da Organização" estarão registrados os objetivos, questões e métricas definidas para a Organização, assim como seus respectivos procedimentos de coleta e armazenamento e análise de dados.

\subsection{A Ferramenta Metrics}

O objetivo da ferramenta Metrics é apoiar a obtenção e o fornecimento dos resultados das medições realizadas de acordo com as necessidades e objetivos dos Planos de Medição da Organização e dos projetos. Além disso, a ferramenta Metrics também fornece apoio para realização e análise das avaliações post morten dos projetos e para o monitoramento da aderência às áreas de processo do CMMI em cada projeto da Organização.

Os cálculos e análises de resultados das medições realizadas são elaborados e apresentados tanto no âmbito da Organização como um todo quanto no âmbito de cada projeto, sendo que os resultados obtidos pela Organização representam a "consolidação" dos resultados obtidos por cada um de seus projetos. As avaliações post morten e das áreas de processo, entretanto, são tratadas apenas especificamente dentro de cada projeto. Após o registro das análises dos resultados a ferramenta automaticamente envia os relatórios gerados para os profissionais que necessitam destas informações. 
Para que os seus objetivos sejam alcançados, a ferramenta Metrics:

(i) calcula as métricas da Organização e de cada projeto separamente;

(ii) auxilia a execução do Processo de Medição e Análise da Organização através da disponibilização de gráficos para visualização dos valores e resultados das métricas calculadas e do apoio à análise dos resultados e geração de relatórios;

(iii) auxilia o gerente de informática da Organização na realização da avaliações post mortem dos projetos, enviando questionários e consolidando e registrando resultados; e

(iv) apoia a análise da evolução da aderência às áreas de processo em cada projeto da Organização, registrando entrevistas, calculando métricas e fornecendo espaço para registro de interpretações, ações de tratamento para não conformidades e inadequações. A Figura 5 apresenta a interface básica da ferramenta.

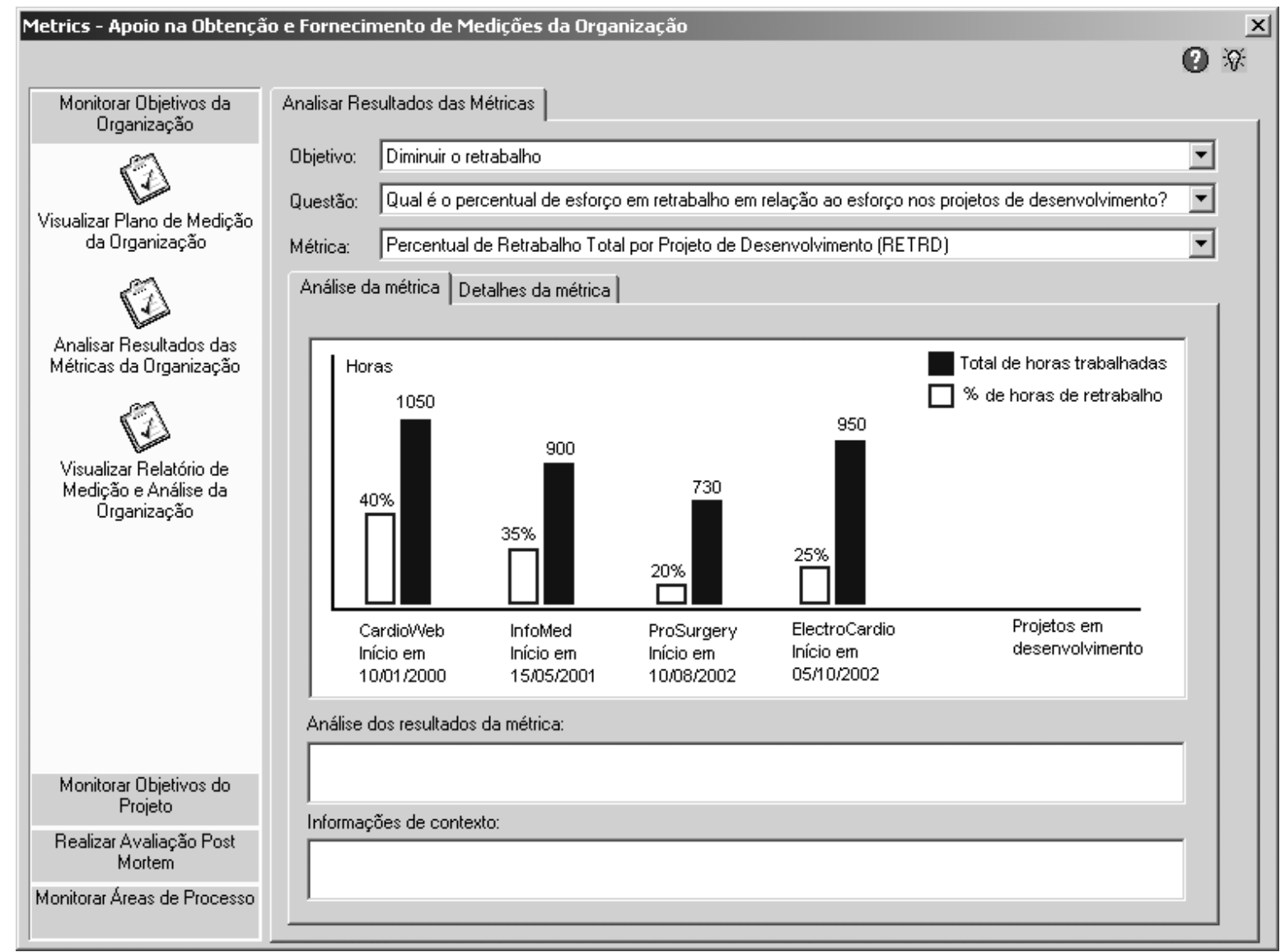

Figura 5 - Tela da sub-atividade "Analisar Resultados das Métricas da Organização"

No lado esquerdo da interface pode-se identificar as atividades "Monitorar Objetivos da Organização", "Monitorar Objetivos do Projeto", "Realizar Avalições Post Mortem" e "Monitorar Áreas de Processo", e suas respectivas sub-atividades. No lado direito identificase a sub-atividade que está sendo realizada pelo usuário. Assim como na ferramenta MedPlan, os ícones localizados abaixo da barra de título permitem a busca e o registro de conhecimento no que diz respeito às atividades do processo e ao conhecimento explícito em relação ao processo de Medição e Análise são disponibilizados através de interface com a ferramenta de Aquisição do Conhecimento Acknowledge [9]. 
$\mathrm{Na}$ atividade selecionada ("Monitorar Objetivos da Organização"), o usuário pode visualizar o Plano de Medição da Organização na sub-atividade "Visualizar Plano de Medição da Organização. A tela da Figura 5 apresenta detalhes da sub-atividade "Analisar Resultados das Métricas", onde o usuário tem acesso aos resultados das métricas que fazem parte do Plano de Medição da Organização.

Para visualizar o resultado de cada métrica o usuário deve selecionar um dos objetivos da Organização, uma das questões associadas a este objetivo e uma das métricas associadas a esta questão. No quadro "Análise Resultado da Métrica" a ferramenta Metrics apresenta os resultados da métrica selecionada nos vários projetos da Organização. O usuário tem espaço então para registrar a análise dos resultados da métrica apresentada e, se desejar, informações de contexto. Na sub-atividade "Visualizar Relatório de Medição e Análise da Organização" a ferramenta apresenta o resultado de todas as métricas da Organização e seus respectivos gráficos acumulativos para que o usuário visualize e imprima, se desejar, e envie aos profissionais responsáveis.

A atividade "Monitorar Objetivos do Projeto" pode ser executada tanto no âmbito da Organização - onde esta pode monitorar especificamente os resultados de medição de cada um de seus projetos, quanto no âmbito dos projetos - onde o gerente de projeto monitora as informações relativas ao seu próprio projeto. Nesta atividade, entretando, as informações relativas aos resultados das métricas apresentados dizem respeito unicamente aos valores obtidos dentro de cada projeto, enquanto que na atividade "Monitorar Objetivos da Organização" os resultados apresentados representam a consolidação, dentro de cada Organização, dos resultados obtidos por cada um de seus projetos.

A atividade "Realizar Avaliações Post Mortem" apóia o usuário na gerência, análise e consolidação dos questionários de avaliação post mortem enviados aos participantes de cada projeto da Organização ao final dos mesmos. Já a atividade "Monitorar Áreas de Processo" permite à Organização auditar a aderência às áreas de processos do CMMI em seus projetos através da análise de avaliações (checklists) e exibição de valores de métricas e seus respectivos gráficos acumulativos.

\section{Considerações Finais}

Um eficaz processo de medição e análise é de fundamental importância para que se obtenha conhecimento sobre o real desempenho de projetos de uma forma geral, mas especialmente de projetos de desenvolvimento de software, onde atualmente é difícil conceber uma gerência com atuação realmente séria sem a utilização de algum tipo de medida [6].

De acordo com McGARRY [8], através da medição é possível obter respostas objetivas a problemas previamente identificados, assim como descobrir e buscar soluções para novos problemas em períodos de tempo bastante curtos. Uma vez que fornece informações reais sobre as Organizações e seus projetos, medir também provê embasamentos objetivos para defesa e justificativa de decisões, assim como melhoram o processo de tomada de decisão de forma geral nas Organizações [8].

Este artigo apresentou uma abordagem para a medição e análise em projetos de desenvolvimento de software fundamentada nos requisitos do CMMI para a área de processo de Medição e Análise e no método GQM. Dentre as principais contribuições da abordagem aqui descrita destacamos:

(i) descrição de um processo de medição e análise baseada no CMMI e no GQM; e

(ii) definição e implementação das ferramentas MedPlan e Metrics, que apoiam a abordagem proposta e encontram-se inseridas no contexto de Ambientes de Desenvolvimento de Software Orientados a Organização. 


\section{Agradecimentos}

Agradecemos ao Conselho Nacional de Desenvolvimento Científico e Tecnológico (CNPq) pelo apoio financeiro ao projeto Ambientes de Desenvolvimento de Software Orientados à Organização.

\section{Referências Bibliográficas}

[1] AHERN, D.M.; CLOUSE, A; TURNER, R., 2001, CMMI Distilled, Addison Wesley.

[2] BASILI. V.; CALDIERA, G.; ROMBACH, H., 1994, "Goal Question Metric Paradigm", In: Encyclopedia of Software Engineering, v.2, pp: 527 - 532.

[3] FLORAC, W., CARLETON, A., 1999, Measuring the Software Process: Statistical Process Control for Software Process Improvement. Addison-Wesley.

[4] GOETHERT, W., HAYES, W., 2001, "Experiences in Implementing Measurements Programs", Software Engineering Measurement and Analysis Initiative, CMU/SEI2001-TN-026

[5] GOETHERT, W., FISCHER, M., 2003, "Deriving Enterprise-Based Measures Using the Balanced Scorecard and Goal-Driven Measurement Techniques", Software Engineering Measurement and Analysis Initiative, CMU/SEI-2003-TN-024

[6] GOLDENSON, D.R., JARZOMBEK, J., ROUT, T., 2003, "Measurements and Analysis in Capability Maturity Model Integration and Software Process Improvement", CrossTalk The Journal of Defense Software Engineering, July.

[7] HOLMES, L., 2002, IT Measurements Practical Advice from the Experts, AddisonWesley

[8] McGARRY, J., CARD, D., JONES, C. et all, 2001, Practical Software Measurement: Objective Information for Decision Makers, Addison-Wesley.

[9] MONTONI, M., MIRANDA, R., ROCHA, A. R.. TRAVASSOS, G. H., "Knowledge Acquisition and Communities of Practice: An Approach to Convert Individual Knowledge into Multi-Organizational Knowledge”, In: Workshop Learning Software Organization, Banff, 2004.

[10] PAULK, M. C., WEBER, C.A., WHITE, D., ZUCCHER, M., 2002, Unpublished data from a study high maturity organizations.

[11] RUBIN, H., 1992, "The Making Measurement Happen Workshop" Proceedings of the $3^{\text {rd }}$ International Conference on Applications of Software Measurements, La Jolla, California, November.

[12] SOLINGEN, R.; BERGHOUT, E., 1999, The Goal/Question/Metric Method: a Practical Guide for Quality Improvement of Software Development, McGraw-Hill.

[13] VILLELA, K., OLIVEIRA, K. M., SANTOS, G., ROCHA, A. R. C., TRAVASSOS, G. H. Cordis-FBC: an Enterprise Oriented Software Development Environment In: Workshop Learning Software Organization, Luzern, 2003

[14] ZUBROW, D., 2001, "The Measurement and Analysis Process Area in CMMI", ASQ Software Quality Newsletter.

[15] ZUBROW, D., 1998, "Measurement With a Focus: Goal-Driven Software Measurement?", CrossTalk The Journal of Defense Software Engineering, September.

[16] CMU/SEI, Capability Maturity Model Integration (CMMI) Version 1.1 - Staged Representation, Carnegie Mellon University, Software Engineering Institute, Pittsburgh, 2002. 